\title{
Effect of Starch Laurate Addition on the Properties of Mango Kernel Starch Films
}

\author{
Laura Gabriela Gurgel de Carvalho ${ }^{a}$, Nívia do Nascimento Marques ${ }^{a}$, Rafael da Silva Fernandes ${ }^{a}$,
} Marcos Antônio Villetti ${ }^{b}$, Men de Sá Moreira de Souza Filho ${ }^{(1)}$, Rosangela de Carvalho Balaban ** (1)

\author{
${ }^{a}$ Universidade Federal do Rio Grande do Norte (UFRN), Natal, RN, Brasil \\ ${ }^{b}$ Universidade Federal de Santa Maria (UFSM), Santa Maria, RS, Brasil \\ ${ }^{c}$ Embrapa Agroindústria Tropical, Fortaleza, CE, Brasil
}

Received: July 22, 2020; Revised: February 25, 2021; Accepted: March 14, 2021

\begin{abstract}
In this work, starch obtained from mango kernel seeds was applied in the preparation of films in combination with its hydrophobic derivatives. Chemical modification of starch was carried out at two different concentrations of vinyl laurate, in dimethyl sulfoxide, under basic catalysis. The degrees of substitution of the starch laurates were 2.4 and 0.3 , respectively. None of the products formed films when dispersed in water. However, blends of starch/starch laurate (degree of substitution $=0.3$ ) formed films after microfluidization of their aqueous suspensions. The blends with up to $15 \%$ wt of starch laurate (degree of substitution $=0.3$ ) resulted in films of higher elongation at break, higher opacity and lower tensile strength and elastic modulus. The microfluidization of biopolymer suspensions was crucial to obtain films with lower water vapor permeability. X-ray diffraction results corroborated to the mechanical properties of the films, showing that the incorporation of starch laurate reduced the starch films crystallinity.
\end{abstract}

Keywords: industrial waste; starch ester; biodegradable films; microfluidization.

\section{Introduction}

The interest in materials from renewable resources with low market value and low environmental impact has grown significantly ${ }^{1,2}$. Starch is one of the most abundant polysaccharides on the planet and can be obtained from many conventional sources, such as cassava, potatoes, rice, corn and wheat ${ }^{3-6}$, and from non-conventional sources, such as plantain, jaca seed, and pea, among others. One nonconventional source less studied is the kernel of seed of mango fruits ${ }^{7-9}$. Due to its low price, biodegradability and renewable origin, starch is one of the raw materials with high potential for food packaging application ${ }^{10-13}$.

The use of kernel seeds of mango fruits (Mangifera Indica L., variety Tommy Atkins) to extract starch is a very interesting proposition in many ways. One of them is the fact that this variety of mango is the most produced and has the largest share in the volume traded in the world, mainly due to its intense coloration, high yields and long-distance transport resistance $^{14-16}$. Brazil occupies a prominent place in the production and exportation of this fruit, producing more than 1 million tons of mango annually ${ }^{14}$. Besides, after industrial processing of mango, portions of the fruit, such as peel and seed, are discarded without proper use. About $22 \%$ of the weight of the mango is due to the seed, from which starch can be obtained ${ }^{17}$. In this sense, the use of mango starch to obtain biodegradable films is very promising, since it enables the reduction of the environmental impacts and leads to economic benefits based on utilization of waste materials ${ }^{18,19}$.

*e-mail: rosangelabalaban@hotmail.com
Although starch films have advantages related to renewal and biodegradation, they have limitations on their mechanical and barrier properties, forming brittle and hygroscopic materials ${ }^{20-23}$. Therefore, the incorporation of additives capable of improving these properties have been investigated ${ }^{22,24-28}$. For instance, formulations based on starch/lipid blends have been studied extensively, generating improvements on the mechanical and barrier properties of films ${ }^{24,29}$. However, lipid-containing films have some disadvantages in the control of film properties, such as: low lipid melting temperature, strong effect of emulsion droplet size distribution, phase separation and strong oxidation tendency over long periods ${ }^{25}$.

The use of nanoparticles, such as starch and cellulose nanocrystals, as reinforcing additives on starch films has been investigated ${ }^{14,15}$. The literature reports positive results, mainly regarding the tensile strength, Young modulus as well as the reduction on water vapor permeability (WVP) $)^{14,15,17}$. However, the use of those nanocrystals significantly reduces the elongation of the films, because of the progressive increase on hydrogen bonding, making the films stiffer and less extensible ${ }^{15}$.

Blending starch with more hydrophobic polymers has been shown to be an interesting combination to produce films for packaging with improved properties ${ }^{30}$. However, because of the intrinsic hydrophilicity of native starch, the poor compatibility between starch and hydrophobic polymers can become a problem to the production of the films ${ }^{31}$. From this perspective, microfluidization, a highpressure homogenization technique, would constitute and alternative route to obtain films from polymers of different 
hydrophilicities, improving miscibility and film properties, including appearance and mechanical properties ${ }^{32,33}$.

In this sense, in this work, starch obtained from mango kernel was hydrophobized by the reaction with vinyl laurate and a new methodology for producing flexible films was elaborated based on microfluidization. The effect of the introduction of different amounts of starch laurate into unmodified starch mango kernel films on the final properties of the products was evaluated. The use of blends composed by starch with starch esters would increase the hydrophobicity of the system, without losing important environmental features.

\section{Experimental}

\subsection{Materials}

Starch (amylose/amylopectin content of 25/75 wt \%) was extracted from mango (variety Tommy Atkins - Mangifera indica L.) kernel (SISGEN: AD3DC70), according to the procedure described by Cordeiro et $\mathrm{al}^{15}$. Sodium hydroxide, hydrochloric acid, hydrogen peroxide solution (30 vol\%), sodium carbonate, potassium carbonate, Tween ${ }^{\circledR} 20$ and dimethyl sulfoxide (DMSO) were purchased from SigmaAldrich. Vinyl laurate (ethenyl dodecanoate) was acquired from Tokyo Kasei. Ethyl alcohol and methyl alcohol were purchased from ISOFAR. Sunflower oil was kindly provided by Embrapa-CE. All the compounds were used without further purification.

\subsection{Determination of weight average molecular weight}

The weight average molecular weight $\left(\bar{M}_{\mathrm{w}}\right)$ of starch was obtained using an Agilent 1260 Infinity Gel Permeation Chromatography (GPC) system equipped with a 1260 Quaternary Pump VL, a PL aquagel-OH MIXED-H $8 \mu \mathrm{m}$ column and a 1260 Infinity Dual Angle Light Scattering $\left(15^{\circ}\right.$ and $\left.90^{\circ}\right)$ detector. The eluent was a $0.15 \mathrm{~mol} / \mathrm{L}$ sodium nitrate aqueous solution containing $0.2 \mathrm{w} / \mathrm{v}$ of sodium azide, previously filtered through $0.45 \mu \mathrm{m}$ pore size cellulose acetate Millipore membranes. The column and the detector operated at the temperature of $40{ }^{\circ} \mathrm{C}$, with a flow rate of $1.0 \mathrm{~mL} / \mathrm{min}$. Calibration was made using dextran standards (American Polymer Standards Corporation). The starch sample $(0.05 \mathrm{mg} / \mathrm{mL})$ was prepared by dissolving the polysaccharide in a $90 / 10 \mathrm{v} / \mathrm{v}$ mixture of dimethyl sulfoxide (DMSO)/water. The biopolymer solution was left under stirring for $24 \mathrm{~h}$ and then it was filtered through $0.5 \mu \mathrm{m}$ pore size polytetrafluoroethylene (PTFE) Millipore membranes. The $\bar{M}_{\mathrm{w}}$ was $3.6 \times 10^{6} \mathrm{~g} / \mathrm{mol}$.

\subsection{Synthesis of starch esters}

Acylation of mango kernel starch (MKS) with different amounts of vinyl laurate (VL) was performed under basic catalysis, according to described in literature ${ }^{19-24}$, but with some modifications. In this case, the objective was to evaluate the effects of different degrees of substitution on the final properties of the starch derivatives and their films. Specifically, $5 \mathrm{~g}$ of mango starch was added slowly to $50 \mathrm{~mL}$ of DMSO, at room temperature $\left(\sim 25^{\circ} \mathrm{C}\right)$, and the biopolymer dispersion was maintained under magnetic stirring for 24 hours. The obtained solution was then transferred to a three-necked flask coupled to a reflux condenser, mechanical stirring, thermometer and a heating mantle, under $\mathrm{N}_{2}(\mathrm{~g})$ atmosphere. Then, the basic catalyst $\left(\mathrm{K}_{2} \mathrm{CO}_{3}\right)$ and vinyl laurate were added in the amounts shown in Table 1 . The system was heated to $110^{\circ} \mathrm{C}$, at a heating rate of $3{ }^{\circ} \mathrm{C} / \mathrm{min}$. The reaction proceeded at $110^{\circ} \mathrm{C}$, for 3 hours. The acetaldehyde gas by-product was removed from the system by absorption in $1 \mathrm{M} \mathrm{NaOH}$, with the aid of a bubbler coupled to the reflux condenser outlet. The system was then cooled to room temperature. Starch laurate 1 (SL1) was recovered by precipitation with methyl alcohol and the purification was carried out by successive washings in a sintered glass Buchner funnel, under vacuum, with methyl alcohol, until the smell of vinyl laurate disappeared. Starch laurate 2 (SL2) was precipitated with water. Afterwards, it was purified by successive washings in a sintered glass Buchner funnel, under vacuum, with water, and subsequently with ethyl alcohol $95 \%$, to remove the unreacted reagents and water. Finally, SL1 was filtrated under vacuum and dried in an oven, at $65^{\circ} \mathrm{C}$, for 24 hours. SL2 was dispersed in water and lyophilized. The yields for SL1 and SL2 were $52 \%$ and $59 \%$, respectively.

\subsection{Determination of the Degree of Substitution $(D S)$}

The DS values were determined using titration method, according to the procedure described in literature ${ }^{27}$. The DS was calculated using the following formula: $\mathrm{DS}=162 \mathrm{M}\left(\mathrm{V}_{\mathrm{o}}-\mathrm{V}\right) / 1000 \mathrm{~W}$, where $\mathrm{V}_{0}$ represents the volume in milliliters of $0.1 \mathrm{~mol} \mathrm{dm}^{-3} \mathrm{HCl}$ solution used for titrating the blank (unmodified starch) and $\mathrm{V}$ is the volume in milliliters of $0.1 \mathrm{~mol} \mathrm{dm}^{-3} \mathrm{HCl}$ solution used for titrating the sample (starch ester, SL1 and SL2). $\mathrm{M}$ is the exact molarity of $\mathrm{HCl}$ solution, $\mathrm{W}$ is the sample weight in grams and 162 represents the molecular weight of the anydroglucose units on starch.

\subsection{Fourier-transform infrared spectroscopy (FTIR) and X-ray diffraction (XRD) analyses of native starch and laurate starches}

Fourier-transform infrared spectroscopy (FTIR) was performed to observe the appearance/disappearance of functional groups that could characterize the chemical modification of starch. The samples were directly placed at the Attenuated Total Reflectance (ATR) accessory and analyzed the wavenumber range from 4000 to $400 \mathrm{~cm}^{-1}$ by the FTIR IRAffinity from Shimadzu.

Table 1. Parameters of vinyl laurate transesterification reaction with starch.

\begin{tabular}{cccc}
\hline Product & Molar ratio of AGU/VL & Catalyst $(\%)^{\mathrm{b}}$ & Purification \\
\hline SL1 & $1: 3$ & 2 & methyl alcohol \\
\hline SL2 & $1: 0.13$ & 2 & water / ethyl alcohol $95 \%$ \\
\hline
\end{tabular}

${ }^{\mathrm{a} a n y d r o g l u c o s e}$ units on starch; ${ }^{\mathrm{b}} \mathrm{wt} \%$ relative to starch. 
In order to observe changes in crystallinity after chemical modification of starch, the biopolymers in powder form were directly placed into a sample holder and analyzed by using a Bruker D2 Phaser diffractometer. The X-ray diffraction (XRD) analyses were carried out using $\mathrm{CuK} \alpha$ radiation $(\lambda=1,54 \AA)$, Ni filter, operated at $30 \mathrm{kV}$ and $10 \mathrm{~mA}$, at the $2 \Theta$ range of $10-90^{\circ}$, with a step size of $0.02^{\circ}$ and scanning speed of $1^{\circ} \mathrm{min}^{-1}$.

\subsection{Films preparation}

Films of mango kernel starch (MKS), SL1, SL2 and their combinations (biopolymer blend films) were produced according to the method described in literature ${ }^{14}$ (Table 2).

In the case of films containing only one biopolymer, $5 \mathrm{~g}$ of MKS or SL1 or SL2 were added to $100 \mathrm{~mL}$ of distilled water under stirring, and heated to $90 / 95^{\circ} \mathrm{C}$, for $30 \mathrm{~min}$, to the gelatinization of starch and suspension of starch esters. Glycerol (25 wt \% with respect to starch) was then added to the system at $60^{\circ} \mathrm{C}$, under stirring, for $15 \mathrm{~min}$. Tween $\AA 20$ (10 $\mathrm{wt} \%$ relative to SL1 or SL2) was added to the SL1 and SL2 suspensions as a emulsifier to improve the dispersion of the hydrophobic laurate chains on the polar aqueous medium ${ }^{34}$.

For the preparation of the biopolymer blend films, aqueous suspensions of starch and its derivatives were prepared separately, according to the conditions shown in Table 2. Starch was suspended in $90 \mathrm{~mL}$ of distilled water and submitted to gelatinization. To SL1 and SL2 preparation, Tween ${ }^{\circledR} 20$ (10 wt\% relative to SL1 or SL2) was added to glycerol ( $25 \mathrm{wt} \%$ with respect to starch) and, afterwards, the starch laurate (amount specified in Table 2) was slowly added to the system, followed by $10 \mathrm{~mL}$ of water to ensure homogenization of the medium. Then, the starch laurate suspension was heated to $90 / 95^{\circ} \mathrm{C}$, for 15 minutes. Finally, starch laurate suspension (SL1 or SL2) was slowly poured into the MKS suspension, under vigorous stirring in Ultra-Turrax (IKA $\left.{ }^{\circledR}-\mathrm{T} 25\right)$, at a rotation of $12,000 \mathrm{rpm}$, for 15 minutes, at $60^{\circ} \mathrm{C}$.

The second stage of development of the films was divided into two routes:
- $\quad$ Route 1: The biopolymer suspensions were transferred to a kitassate, which was sealed and coupled to a vacuum pump, to remove air incorporated during mixture. The process took an average of 30 minutes, with constant heating $\left(60^{\circ} \mathrm{C}\right)$, in order to avoid cooling during the vacuum application.

- $\quad$ Route 2: The biopolymer suspensions were maintained under magnetic stirring overnight, to ensure a good interaction between the biopolymer chains. Afterwards, they were homogenized through a microfluidization processor (Microfluidizer ${ }^{\circledR}$ - Processor M-110EM). The treatments used a mesh of $200 \mu \mathrm{m}$, varying the pressure (5000 and 8000 psi), as described in Table 2. The mixtures went to the rotary evaporator (Buchi Switzerland vacuum controller V-80), under pressure of $260 \mathrm{mbar}$, for $40 \mathrm{~min}$, at $60^{\circ} \mathrm{C}$, for removal of air incorporated in the microfluidization process.

For both routes, after degassing, the mixtures were poured into a glass plate, previously coated with a polyester film (Mylar $\left.{ }^{\circledR}\right)$. The casting process was carried out with the aid of a $1.5 \mathrm{~mm}$ thick stainless-steel bar, which provided better spreading and uniformity of the mixture. The plates were left in a climate-controlled room with controlled temperature and relative humidity of, respectively, $23 \pm 2{ }^{\circ} \mathrm{C}$ and $50 \pm 3 \%$, for $48 \mathrm{~h}$. Only the compositions on Route 2 were able to form films with homogeneous aspect. Then, only the films obtained from Route 2 were able to further characterization.

\subsection{Roughness of films}

For surface roughness determination, the films were analyzed using a digital roughness meter (Taylor-Hobson Surtronic 3, Barcelona, Spain). Each sample was fixed with the aid of a double-sided tape on a test table and the reading considered was the arithmetic mean ( $\mathrm{Ra}$ ) between the peaks and valleys traversed by the active tip of the device, with a measuring path of $0.25 \mathrm{~mm}$. Three readings were taken in different areas on the surface for each sample.

Table 2. Description of composition and conditions of treatment of the films.

\begin{tabular}{|c|c|c|c|c|c|c|c|c|}
\hline \multirow[b]{2}{*}{ Treatments } & \multicolumn{6}{|c|}{ Components } & \multirow[b]{2}{*}{ Pressure $(\mathrm{psi})^{\mathrm{d}}$} & \multirow[b]{2}{*}{ Route } \\
\hline & Water $(\mathrm{mL})$ & MKS (g) & SL1 or SL2(g) & glycerol (g) ${ }^{\mathrm{b}}$ & Tween $(\mathrm{g})^{\mathrm{c}}$ & $\begin{array}{c}\text { Sunflower } \\
\text { oil(g) }\end{array}$ & & \\
\hline SL1 $100^{\mathrm{e}}$ & 100 & - & 5 & 1.25 & 1.5 & - & - & 1 \\
\hline MKS-SL1 50/50 & 100 & 2.5 & 2.5 & 1.25 & 1.5 & 0.6 & - & 1 \\
\hline SL2 100 & 100 & - & 5 & 1.25 & 0.5 & - & - & 1 \\
\hline MKS-SL2 50/50 & 100 & 2.5 & 2.5 & 1.25 & 0.25 & - & - & 1 \\
\hline MKS-SL2 5 & 100 & 5 & 0.25 & 1.25 & 0.025 & - & - & 1 \\
\hline MKS 5000 & 100 & 5 & - & 1.25 & - & - & 5000 & 2 \\
\hline MKS 8000 & 100 & 5 & - & 1.25 & - & - & 8000 & 2 \\
\hline MKS-SL2 5/5000 & 100 & 5 & 0.25 & 1.25 & 0.025 & - & 5000 & 2 \\
\hline MKS-SL2 5/8000 & 100 & 5 & 0.25 & 1.25 & 0.025 & - & 8000 & 2 \\
\hline MKS-SL2 10/5000 & 100 & 5 & 0.5 & 1.25 & 0.05 & - & 5000 & 2 \\
\hline MKS-SL210/8000 & 100 & 5 & 0.5 & 1.25 & 0.05 & - & 8000 & 2 \\
\hline MKS-SL2 15/5000 & 100 & 5 & 0.75 & 1.25 & 0.075 & - & 5000 & 2 \\
\hline MKS-SL2 15/8000 & 100 & 5 & 0.75 & 1.25 & 0.075 & - & 8000 & 2 \\
\hline
\end{tabular}

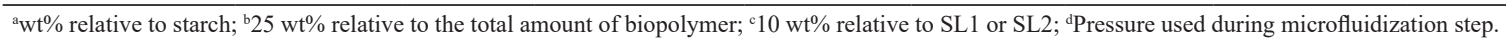
${ }^{\mathrm{e}}$ Compositions using SL1 did not form films or did not form films capable of being characterized. 
Measurements were made in triplicate ( 3 specimens for each film formulation).

\subsection{Opacity}

Film opacity was determined in quadruplicate, following the methodology described in literature ${ }^{35}$. Film strips measuring $2 \mathrm{~cm} \times 5 \mathrm{~cm}$ were placed on the internal side of a Varian Cary $50 \mathrm{UV}-$ Vis spectrophotometer cell, perpendicularly to the light beam. The absorbance spectra (400-800 nm) of film samples were recorded and opacity was defined as the area under the recorded curve (estimated by the linear trapezoidal rule) and expressed as absorbance units $\mathrm{x}$ nanometers (wavelength) per millimeter (film thickness) (A. $\mathrm{nm} \cdot \mathrm{mm}^{-1}$ ).

\subsection{Water vapor permeability (WVP)}

The water vapor permeability (WVP) determination was based on the method E96/E96M-16 $6^{36}$, with at least five replicates. The measurements were made by using $1 \mathrm{~mL}$ distilled water inside permeation cells (inner diameter, $24 \mathrm{~mm}$; height, $10 \mathrm{~mm}$ ) and silica gel as the desiccant material, in an Arsec DCV040 desiccator with air circulation, at $24^{\circ} \mathrm{C}$. Eight measurements were made for each sample within the period of $24 \mathrm{~h}$. Prior to the tests, the sample thicknesses were measured with a digital micrometer (Myutoyo - quantumike IP65). The water vapor permeability (g.mm.Pa $\left.{ }^{-1} \cdot h^{-1} \cdot m^{-2}\right)$ was defined according to Equation 1:

$W V P=W V P R \cdot T / \Delta P$

Where, "WVPR" is the water vapor permeability rate $\left(\mathrm{g} \cdot \mathrm{m}^{-2} \cdot \mathrm{h}^{-1}\right)$ through the film, "T" is the thickness $(\mathrm{mm})$ of the sample and " $\Delta \mathrm{P}$ " represents the difference in partial pressure $(\mathrm{Pa})$ of water vapor between the faces of the film.

\subsection{Tensile tests}

Tensile properties of $125 \mathrm{~mm} \times 12.5 \mathrm{~mm}$ film strips were measured according to the D882-12 standard $^{37}$, with at least five replicates. For that purpose, a Universal Testing Machine (Emic DL-300), equipped with a load cell of $100 \mathrm{~N}$, initial grip separation of $100 \mathrm{~mm}$, and crosshead speed of $50 \mathrm{~mm} / \mathrm{min}$ was employed. Prior to tests, the sample thicknesses were measured with a digital micrometer (Myutoyo - quantumike IP65) and stored for $48 \mathrm{~h}$, under controlled conditions of temperature $\left(23 \pm 2^{\circ} \mathrm{C}\right)$ and relative humidity $(45 \pm 5 \%)$, kept through a saturated saline solution of potassium carbonate.

\subsection{XRD of films}

X-ray diffraction analyzes were performed to verify the retrogradation tendency (recovery of crystallinity) of starch films, as well as the influence of the addition of starch laurate on the morphology of these materials. The films were analyzed on a Bruker D8 ADVANCE diffractometer, using copper radiation $(\mathrm{CuK} \alpha, \lambda=1.54 \AA)$. Scans were performed at a $10 \mathrm{~mA}$ current and $30 \mathrm{kV}$ voltage, using a Lynxeye detector, with a step size of $0.02^{\circ}$ and $0.1 \mathrm{~s}$ acquisition time. The analysis was performed with an angle of $2 \theta$, from 10 to 70 degrees.

\subsection{Statistical analysis}

Values were expressed as mean \pm standard variation. Comparisons between groups were performed using ANOVA and with Tukey's multiple comparison test, performed with Graph Prism 7.0, GraphPad Software Inc., La Jolla, CA, USA.

\section{Results and Discussion}

\subsection{Characterization of biopolymers}

The reaction mechanism for starch laurate formation involved three steps. Initially, an alkoxide is formed by the reaction of the polysaccharide with the base. Then, the carbonyl group of the fatty derivative is attacked by the alkoxide, giving rise to a tetrahedral intermediate. Finally, the leaving group (acetaldehyde) is eliminated and starch laurate is formed ${ }^{19,38}$, according to the mechanism indicated in Figure 1.

The SL1 formed brownish gels which became lighter after precipitation of the product and washing with methyl alcohol. The SL2 product, on the other hand, remained soluble in the solvent (DMSO), since the proportions employed among the reagents tend to generate a low degree of substitution ${ }^{26,41}$. The precipitation and purification of SL2 with methyl alcohol was unsuccessful, giving an unclear product with a strong smell of vinyl laurate. Literature states that highly substituted starch laurates are often purified with methyl alcohol ${ }^{4}$. However, starch laurates with low degree of substitution are better precipitated and purified with water and ethyl alcohol ${ }^{39}$. The use of water and ethyl alcohol 95\% to precipitate and purify SL2 led to a higher yield of a clear product without the characteristic vinyl laurate smell. The degrees of substitution determined by titration for SL1 and SL2 were 2.4 and 0.3, respectively, which are in agreement with the behavior observed during the purification step. After drying, the products were isolated as clear and yellowish solids.

The chemical modification of starch by reaction with vinyl laurate was evidenced by IR spectra (Figure 2), with the appearance of a band at $1740 \mathrm{~cm}^{-1}$ for SL1 and SL2, attributed to the $\mathrm{C}=\mathrm{O}$ elongation vibration of the saturated ester. The higher intensity observed to $\mathrm{C}=\mathrm{O}$ of SL1 (Figure 1c) spectrum may be related to its higher degree of substitution ${ }^{40,41}$. A band centered at $3350 \mathrm{~cm}^{-1}$ can be related to $\mathrm{O}-\mathrm{H}$ stretching vibration of the polysaccharide units and even to some water trapped into the samples. The high intensity of the band at about $1150 \mathrm{~cm}^{-1}$ (compatible to C-O stretching of esters) on the SL1 spectrum, can be related to its high degree of esterification. The absorptions at 2924 and $2854 \mathrm{~cm}^{-1}$ can be ascribed respectively to $\mathrm{C}-\mathrm{H}$ asymmetric and symmetric stretching of both anydroglucose units and laurate portions in the modified starch ${ }^{42,43}$. Starch showed an intense band at $1001.89 \mathrm{~cm}^{-1}$, attributed to C-O stretching of the polysaccharide repeat units. SL2, the copolymer with a lower degree of substitution ( $\mathrm{DS}=0.3$ ), exhibited an enlargement of the $\mathrm{C}-\mathrm{O}$ stretching band, with two absorptions at 999.37 and $1017 \mathrm{~cm}^{-1}$, whereas SL1 (DS = 2.4) underwent a shift on the band to $1040 \mathrm{~cm}^{-1}$. These changes observed between 1100 and $900 \mathrm{~cm}^{-1}$ are typical of starch changing from semicrystalline to amorphous state ${ }^{44,45}$.

The XRD patterns of mango kernel starch (MKS) and starch esters are shown in Figure 3. Diffractogram of starch showed 


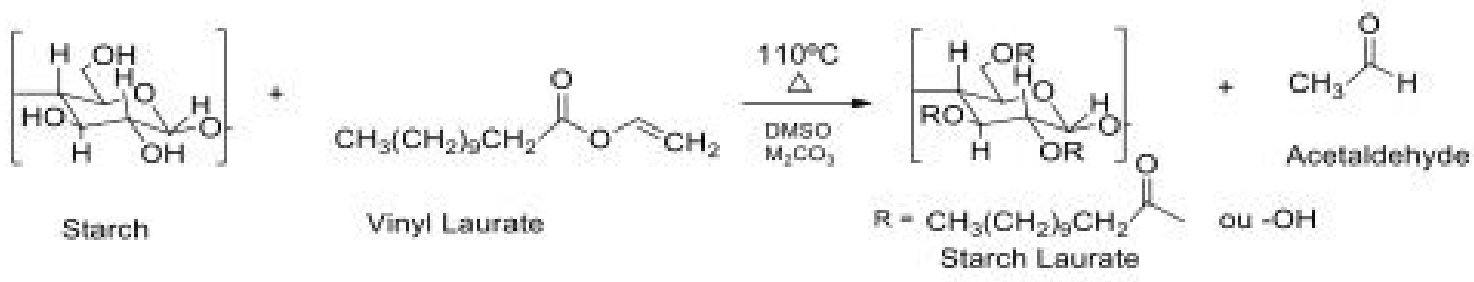

Step 1: Alkoxide is formed by the reaction of the polysaccharide with the base.

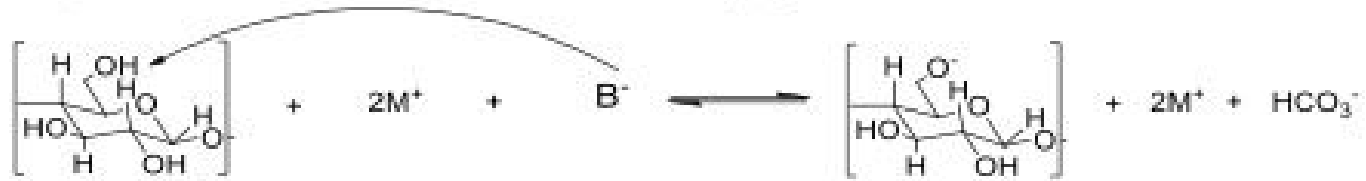

Step 2: Carbonyl group is attacked by the alkoxide.

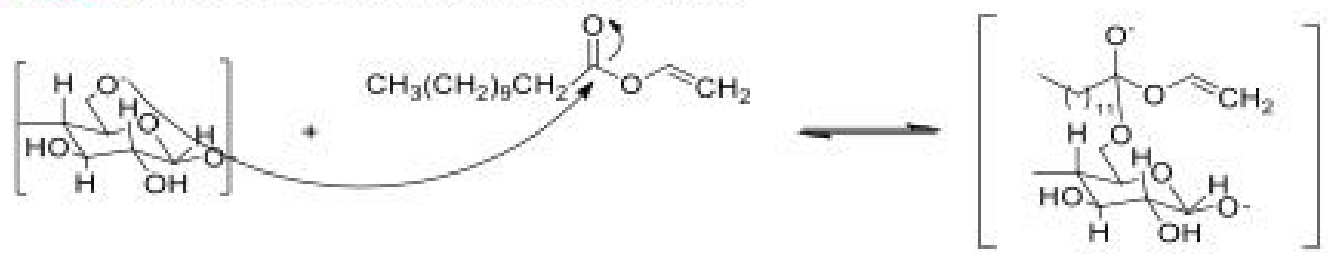

Tetrahedral intermediate

Step 3: Group acetaldehyde is eliminated and starch laurate is formed.
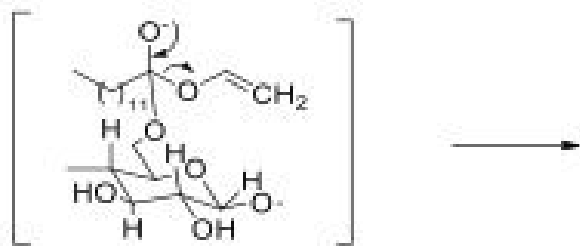

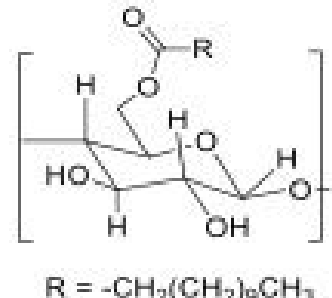

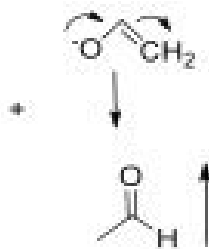

Figure 1. Representation of the steps of starch esterification mechanism with vinyl laurate.

four main peaks at $15^{\circ}, 17^{\circ}, 18^{\circ}$ and $23^{\circ}(2 \theta)$, corresponding to the type A crystallinity pattern. Only a broad amorphous peak was detected in the X-ray pattern of SL1. Clearly, the crystal region of native starch was damaged, indicating that inter- and intramolecular hydrogen bonds in starch molecules were broken during the chemical modification process. However, the copolymer with a low degree of substitution exhibited two diffraction peaks $\left(13^{\circ}\right.$ and $\left.20^{\circ}\right)$, giving a characteristic V-type pattern. The V-type crystallinity of SL2 could originate from the single-helical structure "inclusion complex" composed of starch laurate chains. These results are in accordance with the properties of corn starch laurate found in previous studies ${ }^{43,46}$.

\subsection{Characterization of films}

Preliminary tests were performed to verify the formation of films with the obtained esters and the main results are summarized in Table 3.

After the unsatisfactory results obtained in Route 1, an attempt to improve the polymeric interactions and homogenization of the medium was made through microfluidization (second route). This methodology was satisfactory, since the films presented a homogeneous, flawless and unbreakable aspect during the removal from the support. The microfluidization step was crucial to ensure the homogeneity of the mixture during drying, improving the interaction between the unmodified starch chains and its ester derivative $\mathrm{e}^{33}$.

The films presented homogeneous appearance in all compositions and pressures used during their preparation. This indicates that the microfluidization process improved the biopolymer dispersion in the aqueous medium. On the 
Table 3. Preliminary tests carried out to verify the formation of films with the obtained products (SL1 and SL2).

\begin{tabular}{ccl}
\hline Treatments & Film & \multicolumn{1}{c}{ Aspect } \\
\hline SL1 100 & No & SL1 was insoluble in the aqueous medium even with the use of surfactant. \\
\hline MKS-SL1 50/50 & Yes & $\begin{array}{l}\text { Film had a soaked aspect, exhibiting cracks and small droplets of gels, which were attributed to the } \\
\text { laurate suspension }{ }^{47} \text {, unable to be characterized. }\end{array}$ \\
\hline SL2 100 & No & It formed only aggregation points, attributed to the aggregated SL2. \\
\hline MKS-SL2 50/50 & Yes & $\begin{array}{l}\text { Film had a dry skin aspect and it could not be removed from the drying support, unable to further } \\
\text { characterization. }\end{array}$ \\
\hline MKS-SL2 5 & Yes & $\begin{array}{l}\text { Film was formed and was easily removed from the drying rack with only a few grain-like structures } \\
\text { on the surface. }\end{array}$ \\
\hline MKS 5000 & Yes & \\
\hline MKS 8000 & Yes & \\
\hline MKS-SL2 5/5000 & Yes & \\
\hline MKS-SL2 5/8000 & Yes & Films presented homogeneous appearance. \\
\hline MKS-SL2 10/5000 & Yes & \\
\hline MKS-SL2 210/8000 & Yes & \\
\hline MKS-SL2 15/5000 & Yes & \\
\hline MKS-SL2 15/8000 & Yes & \\
\hline
\end{tabular}

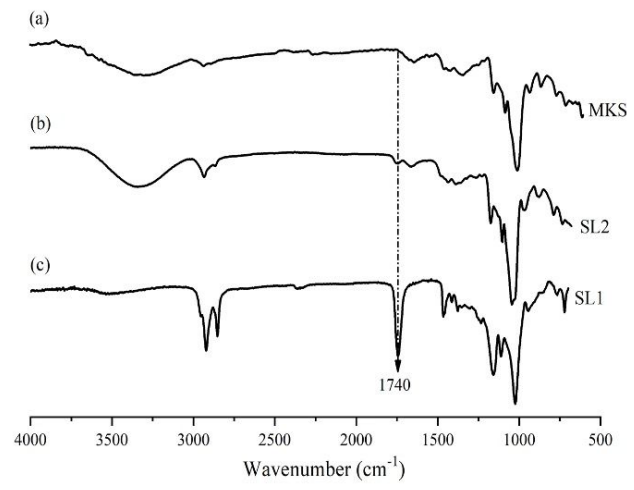

Figure 2. Infrared spectra of MKS (a), SL2 (b) and SL1 (c).

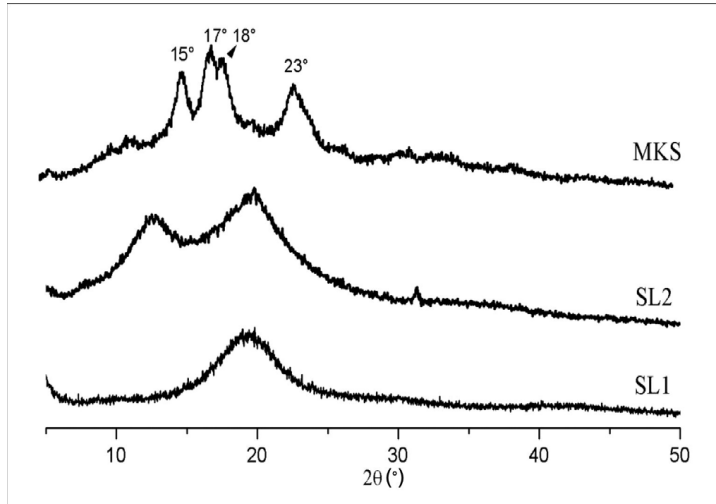

Figure 3. XRD patterns of MKS and its starch esters.

other hand, films prepared by Fakhouri et al. ${ }^{1}$ exhibited white spots when lauric acid was directly added to starch suspensions ${ }^{1}$.

Control group films (MKS 5000 and MKS 8000) had a statistically smaller thickness $(p<0.05)$ than the films with starch laurate. Among the films containing starch ester, no statistically significant differences were observed (Figure 4a). Probably the increase in the thickness of the films containing SL2 is related to different packaging between starch and SL2 macromolecules. In this case, part of the hydrogen bonds made by the starch molecules (films without SL2) are replaced by additional van der Waals interactions between the hydrophobic laurate chains.

In general, the roughness of the films was statistically different for each percentage of added starch laurate, so that the higher the percentage, the higher the roughness values (Figure $4 \mathrm{~b}$ ). The results indicated that the starch laurate modified the packaging of macromolecules during the microfluidization and drying process, causing difference in roughness. However, the films remained with good appearance and homogeneity.

The different pressures used during microfluidization had no significant influence on the average film thickness values. On the other hand, for the roughness of the materials, the pressure was significant for the samples containing 10 and $15 \%$ of starch laurate. This may have occurred because increased pressure promoted greater orientation and better packaging of the molecules, thus changing the topographic characteristics of the films, without changing their thickness.

The visual appearance of films for food packaging such as gloss, color and transparency can affect consumer acceptability and even food quality. In this sense, the influence of the SL2 addition on the opacity of the films was evaluated ${ }^{48}$. The opacity values of all studied films were obtained on a UV-vis spectrometer, using visible light wavelength in the range of 400 to $800 \mathrm{~nm}$. Thus, the opacity was related to the visible light transmission by the films, and the higher the opacity, the lower the visible light transmission, consequently, the lower the transparency.

The films prepared with SL2 displayed a statistically significant difference in opacity in relation to the control group films (MKS 5000 and MKS 8000) from 10\% of SL2 on (Figure 3). This behavior suggests that the SL2 with hydrophobic laurate chains occupy the interchain spaces of the starch matrix, reducing the voids and consequently the transparency of the films $s^{1,26,38}$.

Control films (without SL2 addition) and films containing $5 \%$ SL2 showed no statistical difference in opacity values. The average opacity value $\left(210.5 \mathrm{~A} \cdot \mathrm{nm} . \mathrm{mm}^{-1}\right)$ for these films was similar to that reported in literature ${ }^{14}\left(228.8\right.$ A.nm.mm $\left.{ }^{-1}\right)$ in their control films (composed by starch and glycerol). 

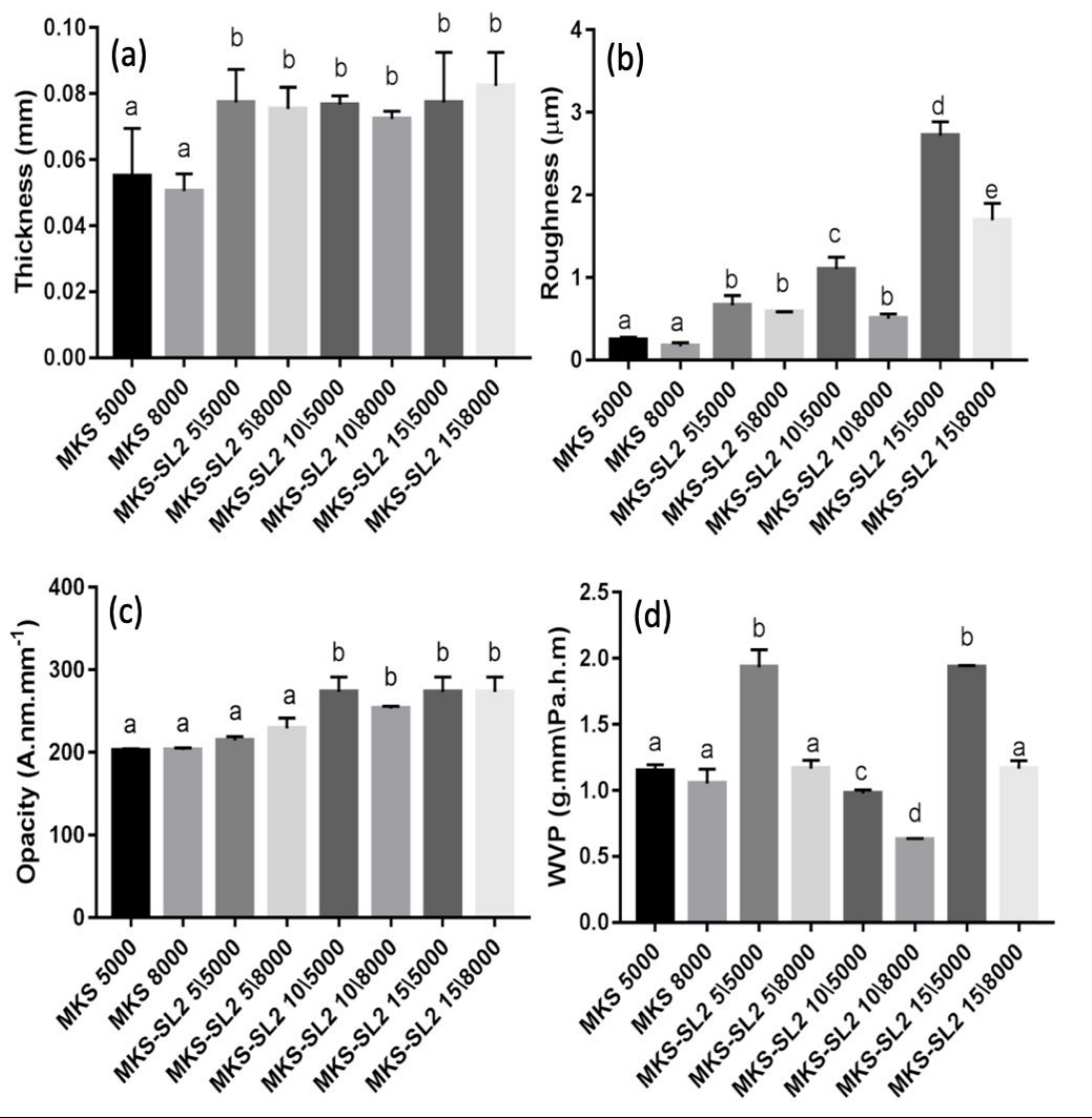

Figure 4. (a) Thickness, (b) roughness, (c) opacity and (d) water vapor permeability of the films. Values sharing a common letter are not significantly different $(\mathrm{p} \leq 0.05)$.

The effects of starch laurate addition and film homogenization pressure variation on the water vapor permeability (WVP) values are illustrated in Figure 4 (d). The lowest WVP values were found for treatments containing $10 \%$ starch laurate (MKS-SL2 10/5000 and MKS-SL2 10/8000), indicating that, at this composition, there was the largest fill of intermolecular spaces in the starch matrix, with homogenization by higher pressure (MKS-SL2 10/8000) being essential to reduce WVP. Farias et al. ${ }^{48}$ stated that the increase in film thickness is also responsible for the increase in WVP. Probably, the microfluidization and the greater hydrophobic character of the films MKS-SL2 10/5000 and MKS-SL2 10/8000 contributed to a lower WVP, even with greater thickness. Similarly, Zahedi et al. ${ }^{47}$ also attributed the decrease of WVP of films based on pistachio globulin protein and fatty acids to the increase on the hydrophobicity.

There are statistically significant differences $(p<0.05)$ in WVP values with increasing pressure for the addition of 5,10 and $15 \%$ of starch laurate. The pressure increase is more significant of WVP results when comparing the samples with 5\% (MKS-SL2 5/5000 and MKS-SL2 5/8000) and 15\% (MKS-SL2 15/5000 and MKS-SL2 15/8000) of SL2. Possibly, $5 \%$ of SL2 was not enough to fill the gaps between starch matrix chains, and the effect was minimized with increased pressure from 5000 to $8000 \mathrm{psi}$, due to a greater packing of the polymeric chains, thus promoting lower WVP values. When the starch laurate concentration was increased from $10 \%$ to $15 \%$, it may have generated the formation of aggregates, leading to an increase of WVP. This result is similar to that reported in literature ${ }^{14}$, that revealed an increase of WVP when the concentrations of starch nanocrystals in starch films was equal to or greater than $15 \%$.

XRD patterns for control films (MKS 5000 and MKS 8000) and with addition of different starch laurate concentrations (MKS-SL2 5/5000, MKS-SL2 5/8000, MKS-SL2 10/5000, MKS-SL2 10/8000, MKS-SL2 15/5000 and MKS-SL2 15/8000) are shown in Figure 5. The diffraction pattern of the control films (without starch laurate) showed peaks at about $17^{\circ}, 20^{\circ}$ and $22^{\circ}$, which are close to type B structures, with a maximum at $17^{\circ}$, associated to crystallization of amylose, due to its linear structure ${ }^{49}$. The peak located at $\approx 32^{\circ}$ can be related to a crystalline organization formed during storage, similarly to the reported by Mendes et al. ${ }^{50}$. Although starch gelatinization is an irreversible process, retrogradation process occurs during drying of the films, leading to the reorganization of the components in crystalline segments, as indicated in the XRD ${ }^{11}$. The addition of SL2 to the formulations gradually decreased the crystallinity of the films. However, a crystalline peak can be observed at approximately $20^{\circ}$ for films with starch laurate, which may be related to $\mathrm{V}_{\mathrm{H}}$-type crystallinity, indicating a higher moisture content present in the formation of the films ${ }^{49}$. 


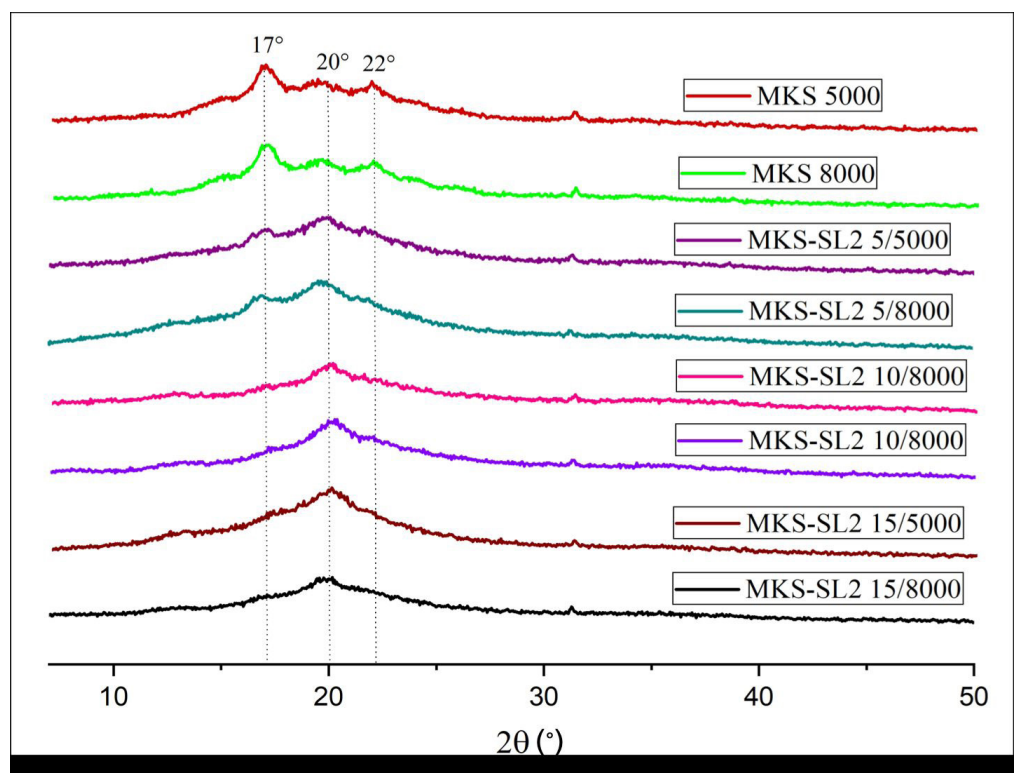

Figure 5. XRD patterns of the different film's formulations.
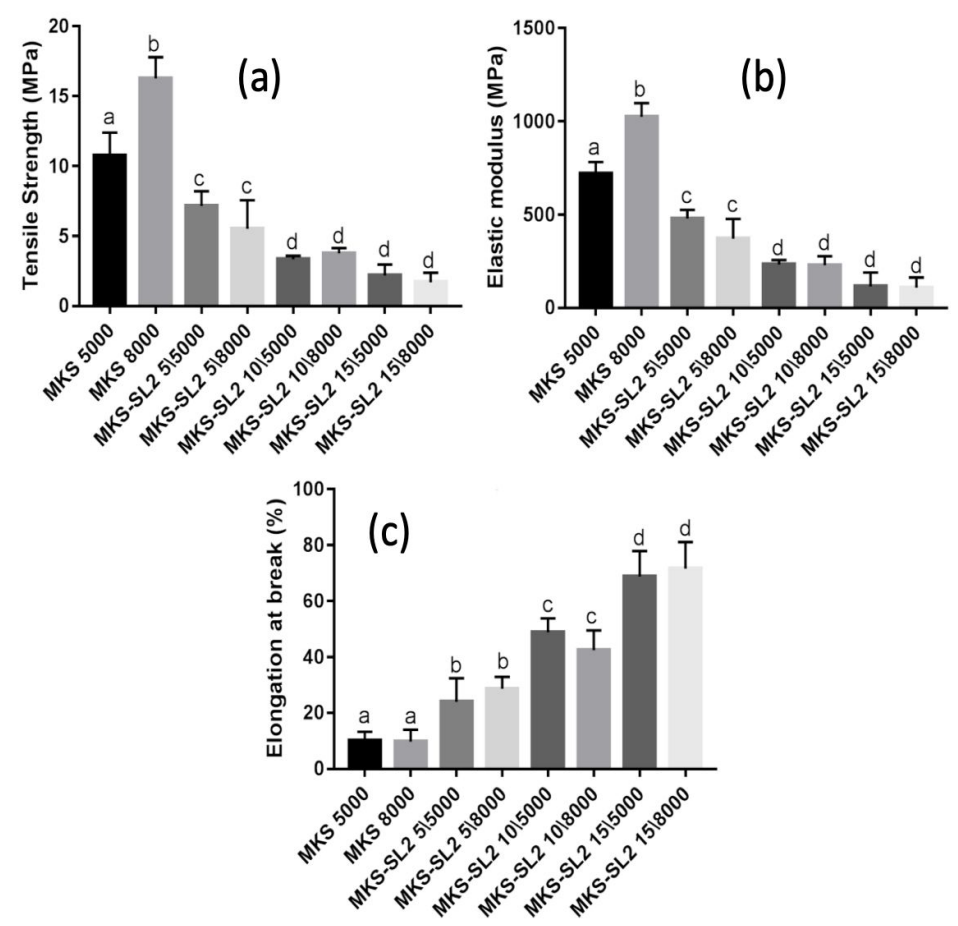

Figure 6. (a) tensile strength, (b) elastic modulus and (c) elongation at break of the films. Values sharing a common letter are not significantly different $(\mathrm{p}<0.05)$.

The mean values of tensile strength, elongation at break and elastic modulus of starch films are shown in Figure 6. These results were calculated from the average data of five specimens for each composition.

The highest rupture stress value (16.26 MPa) was observed for MKS 8000 , suggesting that the high pressure microfluidization process increased the interaction of polymeric chains during film drying, helping the reestablishment of hydrogen bonds, which gave rise to crystalline regions, responsible for the hardness and strength of starch structures ${ }^{11}$. On the other hand, the lowest tensile strength values were found for samples with $10 \%$ and $15 \%$ starch laurate, with no statistically significant difference $(\mathrm{p}<0.05)$ between MKS-SL2 10/5000, MKSSL2 10/8000, MKS-SL2 15/5000 and MKS-SL2 15/8000. One possible explanation for this decline is that the long hydrophobic laurate chains (C12) distributed along the starch derivative (SL2) can decrease the hydrogen bonds interactions among starch chains, which would lead to reduced film resistance (Figure 6a). 
As also shown in Figure 6b, starch laurate insertion significantly reduced the elastic modulus of the films, reaching statistically equivalent values from $10 \%$ of starch laurate, corroborating with the tensile stress results.

Increasing SL2 content resulted on a decreased tensile strength and modulus, and increased elongation at break, indicating a plasticizing effect by SL2 (Figure 6c), as observed by Rodrigues et al. ${ }^{29}$ for tamarind kernel xyloglucan with sesame seed oil, and by Nur Fatin Nazurah \& Nur Hanani ${ }^{51}$ for carrageenan films with oils. Probably, the gradual increase in the amount of SL2 promoted the increase of amorphous regions by filling the interstitial spaces among starch macromolecules, thus significantly reducing the network restructuring through hydrogen bonding ${ }^{34,52}$.

Fakhouri et al. (2009) $)^{1}$ observed an increase in the elongation from 2.5 to $5 \%$ when the lauric acid was added in the concentration range of 5 to $50 \%$ to films based on lipophilic maize starch and gelatin ${ }^{1}$. In our work, the elongation of starch films was increased from about 30 to $80 \%$ by addition of starch laurate in the range of concentration 5 to $15 \%$. This improved performance can be attributed to changes in the polymer chains interactions. In the presence of starch laurate, the retrogradation of starch is inhibited by the long and hydrophobic laurate chains, that decrease the hydrogen bonds between starch chains leading to increase of biopolymer mobility, corroborating with XRD results.

Anyway, the films containing 5 and $10 \%$ SL2 presented the higher and the minimal, respectively, strength acceptable value for food packaging purposes $(4 \mathrm{MPa}$, according to Tajeddin, Rahman, \& Abdulah ${ }^{53}$ ) and higher elastic modulus than low-density polyethylene (ASTM D88-12, 2002 $2^{37}$ ). Furthermore, all films presented much higher elongation than the lowest acceptable value of $10 \%$ for food packaging ${ }^{54}$.

\section{Conclusion}

The applied strategy of insertion of vinyl laurate hydrophobic chains on starch at high DS (2.4) did not give homogeneous mixtures with water for production of films with profitable properties for food packages, even in the presence of surfactants. However, the starch derivative with DS of 0.3 proved to be a good additive in starch films, in formulations up to $15 \mathrm{wt} \%$ of SL2 (relative to starch). Microfluidization proved to be a crucial step to ensure the homogeneity of the mixture during drying, improving the interactions of SL2 with the unmodified starch chains. The increase in pressure during the microfluidization stage resulted in films with less roughness and lower water vapor permeability. The films containing SL2 showed greater thickness, roughness and opacity, compared to the control films (films without SL2). The addition of SL2 was also essential to obtain starch films of higher elongation values, which increased almost eight times (by adding 15\% of SL2). The results obtained in this work show a promising applicability of starch laurate to improve the physical-chemical properties of starch films, that could be used in substitution to synthetic materials in food packages. In addition, the use of starch from a non-eating source and by-product of agribusiness (mango kernel) in industrial products gives the opportunity of a valorization of industrial waste. However, it is still necessary to adjust the composition of the proposed films to improve the tensile strength and elastic modulus to effectively replace materials from non-renewable sources.

\section{Acknowledgements}

The authors are thankful to Petróleo Brasileiro S.A. Petrobras RJ (SAP 4600580022) and Conselho Nacional de Desenvolvimento Científico e Tecnológico (CNPq Grant 304339/2019-9) for their financial support. This study was financed in part by the Coordenação de Aperfeiçoamento de Pessoal de Nível Superior - Brasil (CAPES) - Finance Code 001. Also, the authors gratefully acknowledge the financial support of the Brazilian Agricultural Research Corporation (EMBRAPA, 12.14.04.002.00.00).

\section{References}

1. Fakhouri FM, Fontes LCB, Innocentini-Me LH, CollaresQueiroz FP. Effect of fatty acid addition on the properties of biopolymer films based on lipophilic maize starch and gelatin. Starke. 2009;61:528-36.

2. Jabeen N, Majid I, Nayik GA. Bioplastics and food packaging: A review. Cogent Food Agric. 2015;1:1-6.

3. Aburto J, Alric I, France TC. Preparation of long-chain esters of starch using fatty acid chlorides in the absence of an organic solvent. Starke. 1999;51:132-5.

4. Aburto J, Hamaili H, Senoc F, Alric I, Borredon E. Free-solvent Synthesis and properties of higher fatty esters of starch - Part 2. Starke. 1999;51:302-7.

5. Pérez S, Bertoft E. The molecular structures of starch components and their contribution to the architecture of starch granules: A comprehensive review. Starke. 2010;62:389-420.

6. Frost K, Johansson E, Shanks R, Goto S, Kirwan GM. Thermoplastic starch films: DOE and O2PLS methodology for optimization and increased understanding of polymer processing. Polym Test. 2013;32:343-52.

7. Bonomo RCF, Santos LS, Fontan RCI, Santos OW, Silva AAL. Gelatinization temperature and acid resistance of jackfruit seed starch Temperatura de gelatinización y resistencia ácida de almidón de semilla de jaca. CYTA J Food. 2009;7:1-5.

8. Manoel D, Silene BSS. Propriedades de barreira e solubilidade de filmes de amido de ervilha associado com goma xantana e glicerol. Polímeros. 2011;21(1):67-72.

9. Shittu R, Lasekan O, Karim R, Sulaiman R. morphological characteristics of two cultivars grown in Malaysia. Starke. 2016;68:1187-95.

10. Jiménez A, Fabra MJ, Talens P, Chiralt A. Edible and biodegradable starch films: A Review. Food Bioprocess Technol. 2012;5:205876.

11. Thakur R, Pristijono P, Golding JB, Stathopoulos CE, Scarlett C, Bowyer M, et al. Effect of starch physiology, gelatinization, and retrogradation on the attributes of rice starch- $\mathrm{i}$-carrageenan film. Starke. 2018;70:1-10.

12. Nisa I, Ashwar BA, Shah A, Gani A, Masoodi FA. Development of potato starch based active packaging films loaded with antioxidants and its effect on shelf life of beef. J Food Sci Technol. 2015;52:7245-53.

13. Heung J, Young S, Duck G, Hyeock M, Jin H. Effect of moisture content on the heat-sealing property of starch films from different botanical sources. Polym Test. 2020;89:1-7.

14. Oliveira AN, Silva APM, Barros MO, Souza Filho MSM, Rosa MF, Azeredo HMC. Nanocomposite films from Mango Kernel or Corn starch with starch nanocrystals. Starke. 2018;7:1-7.

15. Cordeiro EMS, Nunes YL, Mattos ALA, Rosa MF. Polymer biocomposites and nanobiocomposites obtained from Mango Seeds. Macromol Symp. 2014;344:39-54. 
16. Pan J, Yi X, Zhang S, Cheng J, Wang Y, Lui C, et al. Bioactive phenolics from mango leaves (Mangifera indica $L$.). Ind Crops Prod. 2018;111:400-6.

17. Silva APM, Oliveira AV, Pontes SMA, Pereira ALS, Souza Filho MSM, Rosa MF, et al. Mango kernel starch films as affected by starch nanocrystals and cellulose nanocrystals. Carbohydr Polym. 2019;211:209-16.

18. Tesfaye T, Johakimu JK, Bruce RBC, Ramjugernath D. Clean Technol. Valorisation of mango seed via extraction of starch: preliminary techno-economic analysis. Clean Technol Environ Policy. 2018;20:81-94.

19. Marques NN, Garcia CSN, Madruga LYC, Villetti MA, Souza Filho MSM, Ito EN, et al. Turning industrial waste into a valuable bioproduct: starch from mango kernel derivative to oil industry. Journal of Renewable Materials. 2019;7:139-52.

20. Menzel C, Andersson M, Andersson R, Vázquez-Guitiérrez JL, Daniel G, Langton M, et al. Improved material properties of solution-cast starch films: effect of varying amylopectin structure and amylose content of starch from genetically modified potatoes. Carbohydr Polym. 2015;130:388-97.

21. Ban W, Song J, Argyropoulos DS, Lucia LA. Improving the physical and chemical functionality of starch-derived films with biopolymers. J Appl Polym Sci. 2006;100:2542-8.

22. Carmo KP, Paiva JMF. Biodegradable films and starch compositions with other materials. Revista Virtual de Química. 2015;7:2377-86.

23. Araújo A, Galvão A, Silva Filho C, Mendes F, Oliveria M, Barbosa F, et al. Okra mucilage and corn starch bio-based film to be applied in food. Polym Test. 2018;71:352-61.

24. Lu Y, Tighzert L. Innovative plasticized starch films modified with waterborne polyurethane from renewable resources. Carbohydr Polym. 2005;61:174-82.

25. Shah U, Gani A, Ashwar BA, Shah A, Ahmad M, Gani A, et al. A review of the recent advances in starch as active and nanocomposite packaging films. Cogent Food Agric. 2015;5:19 .

26. Winkler H, Vorwerg W, Wetzel H. Synthesis and properties of fatty acid starch esters. Carbohydr Polym. 2013;98:208-16.

27. Xie W, Wang Y. Synthesis of high fatty acid starch esters with 1-butyl-3-methylimidazolium chloride as a reaction medium. Starke. 2011;63:190-7.

28. Nawab A, Alam F, Haq MA, Lutfi Z, Hasnain A. Mango kernel starch-gum composite films: Physical, mechanical and barrier properties. Int J Biol Macromol. 2017;98:869-76.

29. Rodrigues DC, Cunha AP, Silva LMA, Rodrigues THS, Gallão MI, Azeredo HMC. Emulsion films from tamarind kernel xyloglucan and sesame seed oil by different emulsification techniques. Food Hydrocoll. 2018;77:270-6.

30. Noivoil N, Yoksan R. Compatibility improvement of poly(lactic acid)/ thermoplastic starch blown films using acetylated starch. J Appl Polym Sci. 2020;138:1-16.

31. Wang X, Huanga L, Zhanga C, Denga Y, Xiea P, Liua L, et al. Research advances in chemical modifications of starch for hydrophobicity and its applications: A review. Carbohydr Polym. 2020;240:1-11.

32. Khan A, Dang K, Chauve G, Bouchard J, Riedl B, Lacroix M. Optimization of microfluidization for the homogeneous distribution of cellulose nanocrystals (CNCs) in biopolymeric matrix. Cellulose. 2014;21:3457-68.

33. Jafari SM, He Y, Bhandari B. Production of sub-micron emulsions by ultrasound and microfluidization techniques. J Food Eng. 2007;82:478-88.

34. Shojaee-Aliabadi S, Hosseini H, Mohammadifar MA, Mohammadi A, Ghasemlou M, Housseini SM. Khaksar. Characterization of $\kappa$-carrageenan films incorporated plant essential oils with improved antimicrobial activity. Carbohydr Polym. 2014;101:582-91.
35. Irissin-Mangata J, Bauduin G, Boutevin B, Gontard N. New plasticizers for wheat gluten films. Eur Polym J. 2001;37:153341.

36. ASTM: American Society for Testing and Materials. ASTM E96/ E96M-16. Standard test methods for water vapor transmission of materials. West Conshohocken: ASTM International; 2002.

37. ASTM: American Society for Testing and Materials. ASTM D882-12. Standard test method for tensile properties of thin plastic sheeting. West Conshohocken: ASTM International; 2002.

38. Muljana H, Picchioni F, Heeres HJ, Janssen LPBM. Green starch conversions: studies on starch acetylation in densified $\mathrm{CO}_{2}$. Carbohydr Polym. 2010;82:653-62.

39. Gao Y, Wang L, Yue X, Xiong G, Wu W, Qiao Y, et al. Physicochemical properties of lipase-catalyzed laurylation of corn starch. Starke. 2014;66:450-6.

40. Hermawan E, Rosyanti L, Megasari L, Sugih AK, Muljana H. Transesterification of sago starch using various fatty acid methyl esters in densified $\mathrm{CO}_{2}$. Int J Chem Eng Appl. 2015;6:152-5.

41. Garg S, Kumar AJ. Characterization and evaluation of acylated starch with different acyl groups and degrees of substitution. Carbohydr Polym. 2011;83:1623-30.

42. Xie W, Shao L, Liu Y. Synthesis of starch esters in ionic liquids. J Appl Polym Sci. 2010;116:218-24.

43. Lu X, Luo Z, Fu X, Xiao Z. Two-step method of enzymatic synthesis of starch laurate in ionic liquids. J Agric Food Chem. 2013;61:9882-91.

44. Vicentini NM, Dupuy N, Leitzelman M, Cereda MP, Sobral PJA. Prediction of cassava starch edible film properties by chemometric analysis of infrared spectra. Spectrosc Lett. 2005;38(6):749-67.

45. Van Soest JJG, Tournois H, De Wit D, Vliegenthart J F G. Short-range structure in (partially) crystalline potato starch determined with attenuated total reflectance Fourier-transform IR spectroscopy. Carbohydr Res. 1995;279:201-14.

46. Geng F, Chang PR, Yua J, Ma X. The fabrication and the properties of pretreated corn starch laurate. Carbohydr Polym. 2010;80:360-5.

47. García-Tejed YV, Leal-Castañeda EJ, Espinosa-Solis V, BarreraFigueroa V. Synthesis and characterization of rice starch laurate as food-grade emulsifier for canola oil-in-water emulsions. Carbohydr Polym. 2018;194:177-83.

48. Farias MG, Fakhouri MF, Ascheri JRL, Carvalho CWP. Caracterização físico-química de filmes comestíveis de amido adicionado de acerola (Malphigia emarginata D.C.). Polímeros. 2012;35:546-52.

49. Amaral LJD, Dias FTG, Zorzi JE, Curz RCD. Obtenção de amidos termoplásticos para a extrusão de pós cerâmicos. Polímeros. 2016;26:1-8.

50. Mendes JF, Paschoalin RT, Carmona VB, Sena Neto AR, Marques ACP, Marconcini JM, et al. Biodegradable polymer blends based on corn starch and thermoplastic chitosan processed by extrusion. Carbohydr Polym. 2016;137:452-8.

51. Nazurah RNF, Hanani ZAN. Physicochemical characterization of kappa-carrageenan (Euchema cottoni) based films incorporated with various plant oils. Carbohydr Polym. 2017;157:1479-87.

52. Rubilar JF, Zúñiga RN, Osorio F, Pedreschi F. Physical properties of emulsion-based hydroxypropyl methylcellulose/ whey protein isolate (HPMC/WPI) edible films. Carbohydr Polym. 2015;123:27-38.

53. Tajeddin B, Rahman RA, Abdulah LC. The effect of polyethylene glycol on the characteristics of kenaf cellulose/low-density polyethylene biocomposites. Int J Biol Macromol. 2010;47:292-7.

54. Khachatryan G, Krzeminska-Fiedorowicz L, Nowak E, Fiedorowicz M. Molecular structure and physicochemical properties of Hylon V and Hylon VII starches illuminated with linearly polarised visible light. Lebensm Wiss Technol. 2014;58:256-62. 